\title{
Developing biological standards for vaccine evaluation
}

\author{
Mark Page ${ }^{*, 1}$, Dianna E Wilkinson', Giada Mattiuzzo', Stacey Efstathiou' \\ \& Philip Minor ${ }^{1}$
}

This perspective describes the process of producing biological reference standards, explains why they are needed, who should use them and in what situations. The aim of this Perspective is to promote their use at an early stage of vaccine development and to make developers aware of the advantages in doing so.

First draft submitted: 4 January 2017; Accepted for publication: 2 May 2017;

Published online: 18 August 2017

The National Institute for Biological Standards and Control produces over 95\% of the world's biological standards. The word standard in this context has specific meaning and importance because of the process by which they are produced. They are not simply a repository of materials but well characterized and rigorously evaluated reagents that serve as a benchmark for the global scientific community. There are several types of physical biological standards but the highest order is that endorsed by the Expert Committee for Biological Standards (ECBS) through the auspices of the WHO. This is a formalized procedure and exacting requirements are set for an International Biological Reference Standard to achieve endorsement [1]

This perspective aims to provide information to those engaged in vaccine evaluation such as manufacturers, clinical trials specialists, epidemiologists and diagnostic kit producers as to the value of using biological standards in their work. Although the information is intended to be generic, the focus is toward viral vaccines to reflect the discipline of the journal but also due to the areas of expertise of the authors.

\section{Why are biological standards required?}

The definition of a 'biological' in WHO documents is "a substance which cannot be fully characterized by physico-chemical means alone, and which therefore requires the use of some form of bioassay" [1]. Essentially, a biological standard is a positive reference sample that is included in a biological assay to ensure that it is functioning correctly. For vaccine evaluation, this would normally be an antibody, antigen or infectivity standard. Biological standards are used in assays that measure the potency, purity and identity of complex biological materials such as vaccines. They also have important applications in the standardization of nucleic acid-based tests, including real-time PCR, and serology assays used in epidemiology and clinical trials to evaluate vaccine efficacy. A biological standard that is common to all assays allows for calibration and harmonization of assay data (Figure 1). This is important when evaluating, for example, the antibody response elicited by a candidate vaccine and for comparing results using different vaccines or immunisation regimens. If the data are reported in microgram per mil-

'Division of Virology, National Institute for Biological Standards \& Control, Blanche Lane, South Mimms, Potters Bar, Herfordshire, EN6 3QG, UK

*Author for correspondence: mark.page@nibsc.org

\section{KEYWORDS}

- antibody • assay development $\bullet$ biological standard $\bullet$ reference materials $\bullet$ unitage $\bullet$ vaccine evaluation 


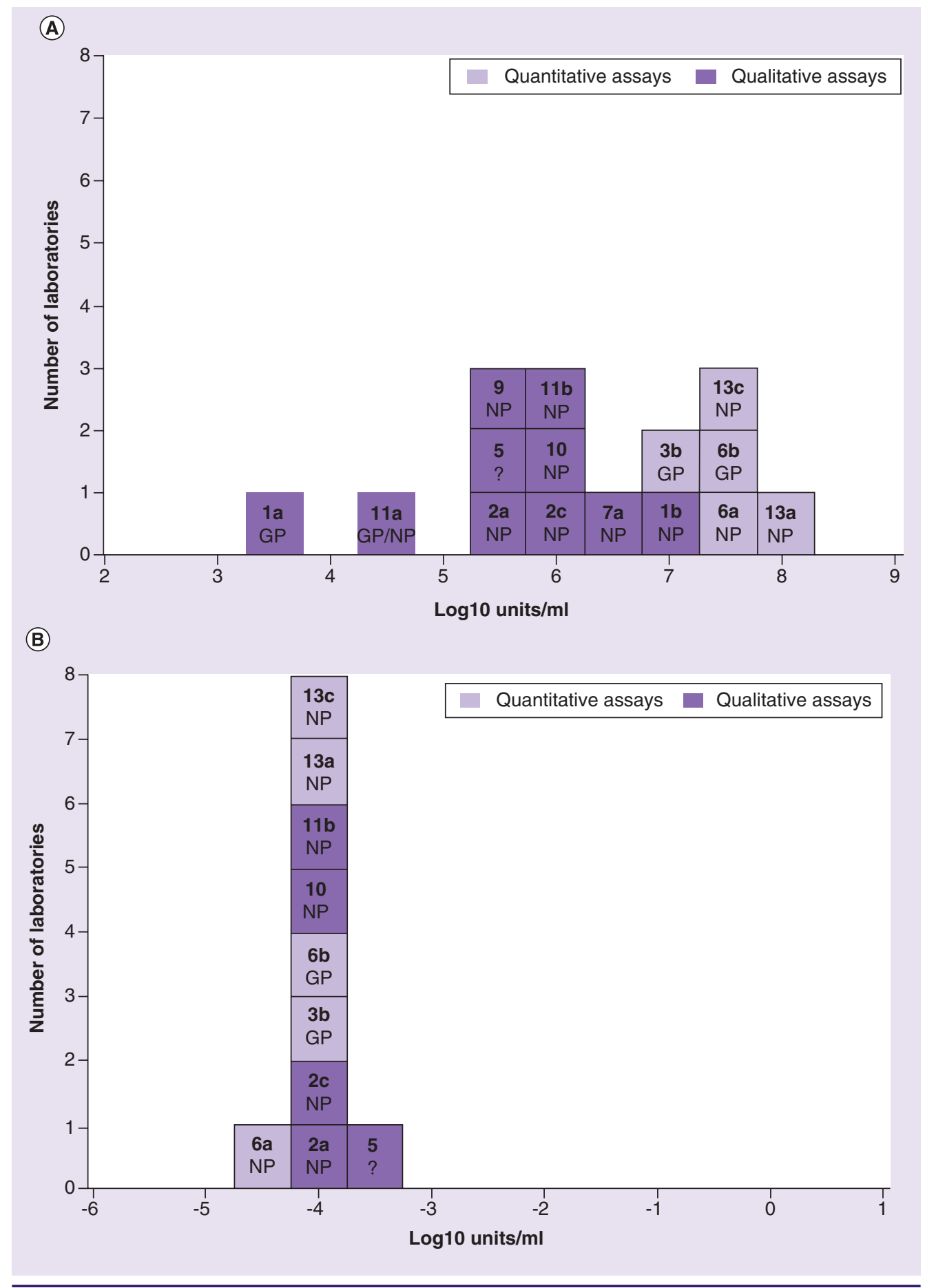

Figure 1. Representative collaborative study data returned to National Institute for Biological Standards and Control for analysis, in this case showing estimated potencies for a candidate NAT standard for Ebola. Histogram of the mean laboratory estimates in quantitative (light blue squares) or qualitative (dark blue squares) NAT assays. The estimated values are shown on the horizontal axis and the number of laboratories is indicated on the vertical axis. The results are reported as Log10 'copies'/ml for quantitative assays and Log10 'NAT'-detectable units/ml for qualitative assay. Each box represents the mean estimate from each laboratory assay and is labeled with the laboratory code and assay target. There is a widespread ( 5 logs) difference in values for the same material observed. 
Figure 1. Representative collaborative study data returned to National Institute for Biological Standards and Control for analysis, in this case showing estimated potencies for a candidate NAT standard for Ebola (cont. from previous page). When data is taken as reported by individual laboratories (A), whereas if the data are reported as a relative potency to the candidate standard, agreement between laboratories is improved to less than 2 logs (B).

Data taken from [2].

liliter of $\operatorname{IgG}$ for example, is the microgram value reported by one assay or laboratory equivalent in its activity (potency) to that reported by another, given that the serum will contain a heterogeneous and nonreproducible mixture of molecules of immunoglobulin? To overcome this dilemma, the biological standard is designated an arbitrary unitage of activity/potency. In the case of WHO International Standards, this is the International Unit (IU). Secondary, tertiary and working standards can be calibrated in terms of the IU. The activity of each sample is then calculated, usually by parallel line analysis, to have a potency relative to the standard. Examples of these are for hepatitis $\mathrm{B}$ and measles vaccines where protective antibody titers are recognized to be $>10 \mathrm{mIU} / \mathrm{ml}$ and $>120$ $\mathrm{IU} / \mathrm{ml}$ respectively in specific immunoassays or virus neutralization assays.

The concept of an arbitrary unitage is important in allowing for appropriate standardization of assay data and should be adopted as early as possible during vaccine evaluation. In this way, a vaccine's, potency, immunogenicity or efficacy may be formally compared between different vaccines and studies and reported in a common format that has relevance and can be interpreted meaningfully when measured in a range of assays.

\section{What are they?}

Ideally, the standard should be an analyte that works in a broad range of different assays and reflects how a test sample would behave in that assay. This is a complex problem and not all standards fit this attribute of commutability [3]. For antibody assays, an ideal starting point is to use the serum or plasma from an individual who has been infected with the relevant pathogen. This material usually has a broad range of antibody specificities that will have a high likelihood of reacting in a wide range of assays including ELISAs of various formats and neutralizing antibody assays. Pooling samples from several individuals can widen the spectrum of antibody reactivities. A number of particular issues can however arise with this approach, primarily the difficulty in obtaining such samples from willing patients who kindly consent to donate a relatively large volume of their blood. Moreover, there might be biosafety restrictions over how to handle the sample in the laboratory. Is containment needed? Can the sample be inactivated such that a reference standard is produced from it and then be suitable to ship around the globe?

Other options are to use antibody samples from vaccinated subjects and immunized animals. Serum or plasma from individuals vaccinated with the vaccine under evaluation may have a restricted specificity especially if it is a subunit vaccine but could have utility for the specific purposes of comparing responses of different immunization regimens. Using animal serum is an option in the absence of other alternatives but in our experience, these have not performed well against human samples [4]. A recent advance that has utility and could address short-term needs is the use of transchromosomal bovines that produce fully humanized antibodies [5]. These have the advantage of supplying large volumes of antibody in a relatively short timeframe but like vaccine antibodies may be limited in use by a restricted antibody repertoire that is dictated by the vaccine composition.

For nucleic acid-based assays, the optimal preparation would be the complete pathogen as this would contain the entire genome and therefore represent the clinical sample very closely depending on the medium it is presented in. In some cases this is not appropriate given that the infectious agent may be a high hazard group (groups 3 and 4) pathogen and therefore would best be inactivated to assure biosafety but also to enable it to be handled in a lower biocontainment level two laboratory. Another method is to use a synthetic approach by packaging the nucleic acid in virus-like particles [6]. This method also controls for the nucleic acid extraction process where plasmid DNA/RNA preparations would not be suitable.

Vaccine reference standards are also prepared that can calibrate assays measuring vaccine potency in terms of titer, antigen content or immunogenicity and provide for quality control 
of manufacturing consistency [7]. Such standards are ideally prepared from inactivated pathogen preparations or bulk vaccine preparations. For some vaccines, quality control is based on the demonstration of consistency in comparison with a product-specific standard which is traceable to a batch shown to be efficacious in clinical trials [8]. There are a variety of assays that are used to measure the vaccine potency either through in vivo studies where the vaccine under test is titrated and the protective titer determined by subsequent challenge with wild-type virus; the titer is determined by comparison to the vaccine standard or by in vitro methods that can measure the antigen content (ELISA-type format).

A standard needs to have a long shelf life and therefore have high stability. Using a standard over many years assures that assays do not drift in their performance by regular checking against the standard or using in-run controls to monitor for any drift over time; these in-run controls can be calibrated against an appropriate standard, preferably the International Standard. Many standards are freeze-dried allowing storage at ambient temperatures and for reconstitution in an appropriate buffer at the time of use.

Secondary or working standards are often needed and produced for national, regional or other organizations with greater quantities of calibrated and accredited material used in batch control and other product development activities, for which the International Standard may not be available due to limited supply [8]. In other words, these secondary standards are used more frequently and help to preserve the longevity of the International Standard. Moreover, such standards are often close to the limit of detection so that assay sensitivity may be tested and monitored. Secondary standards are likely to be replaced more frequently but still should be stable and calibrated to the International Standard through a collaborative study (see later "how are they made?") and should be produced in a similar manner to that required for the International Standard.

\section{When should they be used?}

It would be advantageous to use antibody standards at the outset of vaccine development such as during the preclinical development stages where animal model investigations that establish both immunity and protective capacity may be determined. Unfortunately, reference materials and standards are not usually available due to the protracted timeline it takes to produce a well-charac- terized standard. The benefits of having a standard at this stage though are that any animal model work undertaken which determines a protective antibody titer may be bridged to clinical trials and some understanding of whether the vaccine can induce antibody responses of sufficient titer in humans can be garnered. This rarely happens in practice however, and recently would have been useful for work on Ebola vaccine candidates in correlating responses between the macaque studies and responses where protection was observed in clinical trials. Surprisingly, the introduction of a standard is somewhat ad hoc and precipitated for the need to provide assays that can measure the consistency of a vaccine lot close to or after licensure. Again this is more of a consequence of the time it takes to produce appropriate standards. However, there is an increasing awareness of the need to have standards in place at an early phase of vaccine development particularly after the recent Ebola and Zika outbreaks where there was an urgent need for accurate diagnosis of the infection through the use of validated and calibrated assays. Inaccurate diagnosis could have huge implications for the affected individual and their consequent health care provision, which may well be a matter of life or death. The WHO have implemented an Emergency Use and Assessment Listing procedure to address this need for accurate diagnosis for emerging epidemics that have been declared a Public health Emergency of International Concern [9]. National Institute for Biological Standards and Control (NIBSC) was requested by the WHO to produce standards for diagnosis and vaccine evaluation for Ebola in its role as a WHO Collaborating Centre to meet this urgent need. A similar request has been made for the Zika virus outbreak in central South America. This reflects the changing face of the requirement for standards to be produced for emergency situations in accelerated timeframes. To respond to these requests NIBSC needs to use its resources optimally and draw on the scientific community and national health authorities to provide source materials and participate in collaborative studies to characterize and establish these standards.

\section{How are they made?}

The process and timelines for producing a standard is illustrated in Figure 2. As stated above, the procedure for producing an international standard is undertaken through the ECBS. The approval to start is given by the ECBS after consideration that the standard is required and justified. The 
source materials are then collected. As mentioned earlier, these arecollected; typically plasma/serum for antibody standards and pathogens/antigens for vaccine standards. The materials are then characterized in a small number $(\sim 3-4)$ of laboratories to confirm their potential as a candidate reference standard. Suitable materials are then compiled into bulk solutions and aliquoted. Part of this process may involve trial fills of ampoules to determine the suitability of the filling process and stability of the function of the finished product. The choice of the final preparation is dependent on its intended use, and several options may be included for analysis in the subsequent collaborative study. Also, careful consideration is given to the long-term stability of the product and the composition of the freeze drying conditions is a key step. Ideally, some accelerated degradation studies at higher than ambient temperatures are undertaken to provide information on the stability of the product.

The critical feature of the process of establishing a standard is a large collaborative study that allows a useful comparison of both intra- and interlaboratory performances to be tested as well as the assays themselves. Initially, laboratories with relevant experience are invited to participate and a questionnaire sent to determine what assays they can perform. This may be preceded by a workshop to define the assay landscape, to design the study protocol and to recruit participants. Feedback is requested on the protocol design for testing of the candidate standards. This usually takes the form of the sample being assayed in triplicate on three separate occasions as a minimum. Importantly, the samples are coded blind so that the participating laboratories do not know their identity and in this way provide an objective analysis. The data from the assays is collected and collated and statistical analysis undertaken. These data are then compiled into a report which is circulated to the participants for amendments, comments and clarification on the findings. If the data allow, the candidate reference standard is identified and a final report submitted to the ECBS. The ECBS meets annually in October to review the collaborative study reports and decides on endorsement of the recommendations. In emergency situations,

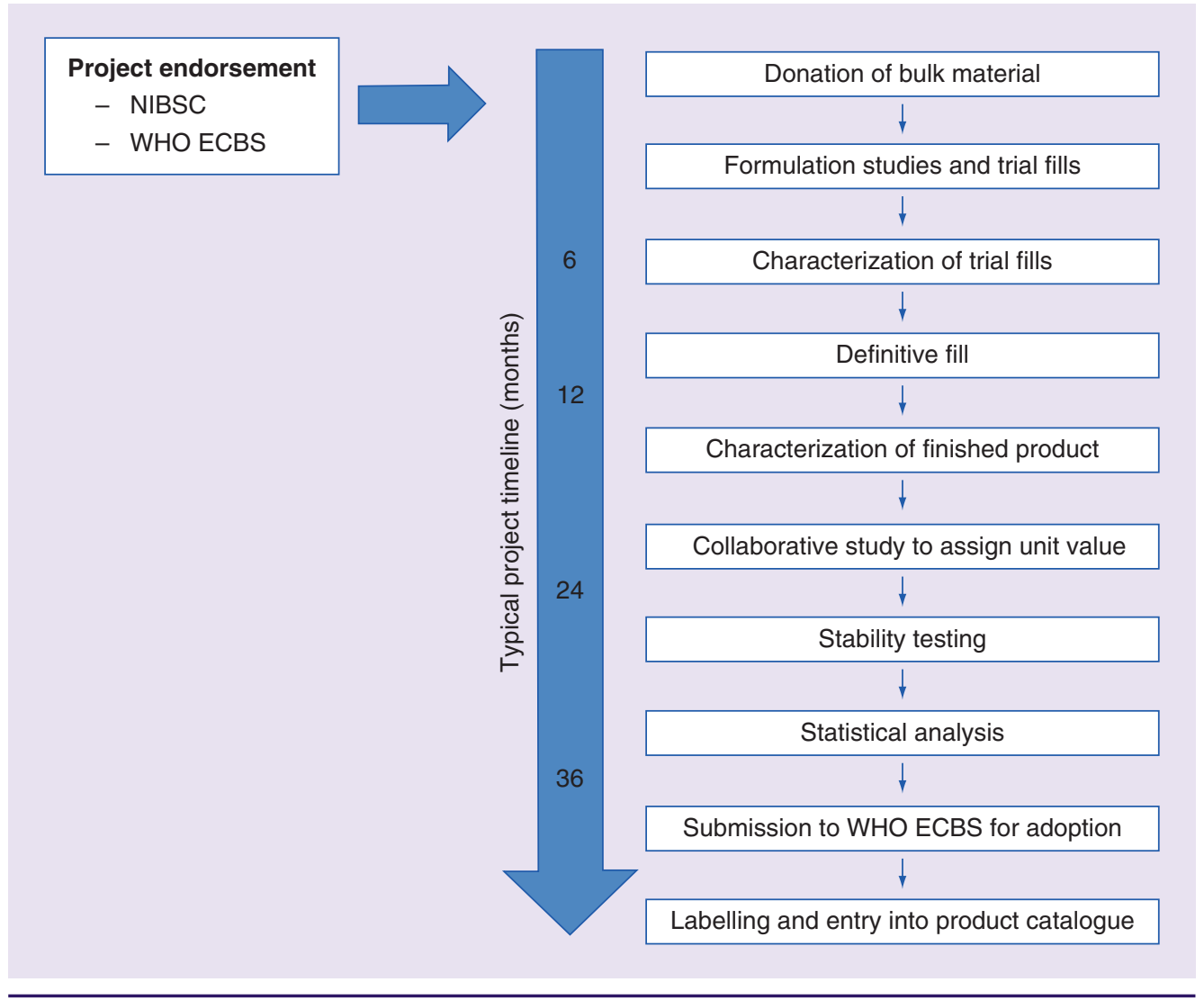

Figure 2. Schematic of processes and timelines for the production of a typical biological standard. 
the ECBS can endorse a standard ad hoc. Once the standard is endorsed, the report is filed on the WHO IRIS website [10]. The standards that NIBSC produces are listed in its catalog [11]; and can be purchased for a small administrative cost.

The storage and stability of a standard is clearly a key aspect given that the ampoules are required for use over an extended timeframe of many years. The storage conditions must therefore be defined to assure that the standard remains stable and retains the same level of activity over time. The standard is granted official status on the basis of its long term stability data which are collected by storage of the ampoules at various temperatures and testing the activity of those samples at regular (initially monthly) intervals. Accelerated degradation studies using the Arrhenius Equation [12] can be done to predict loss of potency by storage at higher temperatures but real time stability data are the best approach. Freeze drying is often used to provide a long shelf life and to facilitate shipping which can be done at ambient temperatures, but this is very dependent on the type of standard and often involves development work to optimize the freeze drying conditions and monitoring of various parameters most critical of which is the moisture content. Further, specialist equipment is needed to freeze dry thousands of ampoules consistently. Frozen liquid standards clearly are easier to produce, but it is critical that the ampoules are maintained at the correct temperatures and this will require the installation of well maintained freezers, which may not be available to all end point users.

\section{Who should use them?}

The intended use of the standards is not restricted and we encourage laboratories that run assays where there is a need for calibration and harmonization with other assays to use a reference reagent. Also, by calibrating a run control in the assay against the standard on a regular basis then this provides a means to monitor assay performance over time which may drift or be modified with use. The intended purpose of the International Standard is to be used to calibrate a secondary reference reagent and not to be used in each run as this will soon exhaust the batch. The supply of the International Standards is limited to two ampoules per year per laboratory for this reason. International biological reference standards are distributed free of charge to national control laboratories and intergovernmental organizations for their intended purpose.

"International biological reference standards are usually available in relatively limited quantities and are intended to be used for the characterization and calibration of secondary preparations, whether of regional, national or more limited status; these secondary preparations are then used routinely" [1].

\section{Future perspective}

There is an increasing need to make standards to address emerging infections, however achieving this in an expeditious manner is problematic given the time it takes to source materials and run collaborative studies. New technologies and platforms may help in this regard but also there is an increasing realization that the scientific community needs to be prepared for any new Public Health Emergency of International Concern (PHEIC). This is being addressed through new initiatives such as the WHO R\&D Blueprint [13] and the Coalition for Epidemic Preparedness Innovations [14].

In an age of increasing use of biological medicines such as monoclonal antibodies and vaccines and their inherent complexity, the need for biological standards continually increases. The requirement for a physical standard with material in a bottle rather than a virtual one cannot be emphasized enough.

\section{Conclusion}

Standards are important reagents that have a well established precedent for assay validation, calibration and harmonization. Their wider use and early adoption in product development will promote accurate diagnosis and assay assurance for vaccine evaluation of clinical trial efficacy and vaccine production consistency.

\section{Financial \& competing interests disclosure}

The authors have no relevant affliations or financial involvement with any organization or entity with a financial interest in or financial conflict with the subject matter or materials discussed in the manuscript. This includes employment, consultancies, honoraria, stock ownership or options, expert testimony, grants or patents received or pending, or royalties.

No writing assistance was utilized in the production of this manuscript.

\section{Open access}

This work is licensed under the AttributionNonCommercial-NoDerivatives 4.0 Unported License. To view a copy of this license, visit http://creativecommons.org/ licenses/by-nc-nd/4.0/ 


\section{EXECUTIVE SUMMARY}

- International Biological Standards are established through a formalized procedure endorsed through the auspices of the WHO.

- Standards permit the calibration and harmonization of assays for comparison of vaccine potency and immunogenicity.

- Standards are assigned an arbitrary unitage to avoid variations in the measured specific activity of the analyte under test.

- Standards are chosen to work across a large range of assays and behave as close to a clinical sample as possible. They should have a long stability and produce in sufficient numbers so that they are available for many years.

- Their early adoption in vaccine evaluation is recommended so that comparative analyses may be undertaken at the preclinical stage and bridged to clinical studies.

- Standards are produced and established through a collaborative study approach in which candidate samples are tested by participating laboratories using their assays. The samples are coded and blinded to the participants. The study data are statistically analyzed and evaluated by an expert committee on behalf of the WHO.

- Optimization of the filling and freeze drying of the standards is a key component to ensure a long shelf life and stability.

- The use of standards is not restricted and all laboratories can benefit in including them in their assays.

\section{References}

Papers of special note have been highlighted as:

- of interest

1 WHO. Recommendations for the preparation, characterization and establishment of international and other biological reference standards. In: WHO

Expert Committee on Biological Standardization. WHO Technical Report Series, no. 932. WHO, Geneva, Switzerland (2006).

- Describes in detail the procedure required for the establishment of an International Standard.

2 Wilkinson DE, Page M, Almond N et al. Expert committee on biological standardization preliminary report WHO collaborative study to assess the suitability of interim standards for Ebola virus NAT assays. WHO, Geneva, Switzerland (2015).

- This report is a useful example of what is involved in a collaborative study for establishing a biological standard.

3 WHO. WHO consultation on commutability of WHO biological reference preparations for in vitro detection of infectious markers. Geneva, Switzerland, 21-25 October 2013.

4 Stephenson I, Heath A, Major D et al. Reproducibility of serologic assays for influenza virus A (H5N1). Emerg. Infect. Dis. 15(8), 1250-1259 (2009).

5 Dye JM, Wu H, Hooper JW et al. Production of potent fully human polyclonal antibodies against Ebola Zaire Virus in transchromosomal cattle. Sci. Rep. 6, 24897 (2016).

6 Mattiuzzo G, Ashall J, Doris KS et al. Development of lentivirus-based reference materials for Ebola virus nucleic acid amplification technology-based assays. PLoS ONE 10(11), e0142751 (2015).

7 WHO guidelines on nonclinical evaluation of vaccines. In: WHO Technical Report Series, No. 927. WHO, Geneva, Switzerland (2005).

8 WHO manual for the establishment of national and other secondary standards for vaccines. WHO, Geneva, Switzerland (2011).

- Details the requirements for the production of secondary and working standards.
9 WHO. WHO emergency use assessment and listing procedures for medical products during public health emergencies (2016). www.who.int

10 WHO IRIS: Home. http://apps.who.int/iris/

- Website link for a large searchable series of reports describing collaborative studies and associated documents around biological standards and guidelines.

11 NIBSC biological reference materials. www.nibsc.org

- $\quad 95 \%$ of the world's standards are available here. A large range of viral and bacterial standards for vaccines evaluation are listed.

12 Kirkwood TB. Predicting the stability of biological standards and products. Biometrics 33(4), 736-742 (1977).

13 WHO. A research and development blueprint for action to prevent epidemics. WHO (2017). www.who.int

14 CEPI. http://cepi.net 